\title{
Electrical signature of chiral magnetic textures in synthetic antiferromagnets
}

\author{
Davide Maccariello, ${ }^{*}$ William Legrand, Nicolas Reyren, Fernando Ajejas, Karim \\ Bouzehouane, Sophie Collin, Jean Marie George, Vincent Cros ${ }^{\dagger}$ and Albert Fert \\ Unité Mixte de Physique, CNRS, Thales, Univ. Paris-Sud, \\ Université Paris-Saclay, 91767 Palaiseau, France
}

\begin{abstract}
By using magnetic multilayers with compensated perpendicular anisotropy and antiferromagnetic coupling between neighboring layers, we show that it is possible to electrically detect the signature of non-collinear magnetization textures in synthetic antiferromagnet systems. With the support of magnetic force microscopy and micromagnetic simulations, we enlighten the phenomena behind the magnetotransport measurements. The observed magnetoresistive response can be phenomenologically described by two competing main contributions: a GMR-like interlayer contribution and an intralayer contribution due to in-plane magnetic moments present in any non-collinear magnetic texture. Relying on both temperature and field-dependence measurements, we show that the details of the magnetic textures can be quantitatively determined through electrical measurements.
\end{abstract}

From the early years of spintronics, synthetic antiferromagnetic (SAF) multilayers have attracted considerable interest for their use in spintronic technologies such as one of the key elements for magnetic sensors or recording heads in hard disk drives. In SAF system, the ferromagnetic (FM) layers are separated by a non-magnetic metallic (NM) spacer having thickness sets at the antiferromagnetic maximum of the indirect interlayer exchange coupling $[1,2]$.

Recently, there is a renewed interest for these magnetic stacks, but in systems with perpendicular magnetic anisotropy (PMA) for their potential application in skyrmions and domain walls based devices [3, 4]. In fact, the Dzyaloshinskii-Moriya (DM) interaction [5-7] rising at the interfaces of the FM/NM stacking can lead to the stabilization of homochiral magnetization configuration i.e. domain walls and skyrmions. In addition, the antiferromagnetic coupling achieved through nonmagnetic metal interlayer gives rise to a negligible stray field which can lead to the room-temperature stabilization of nm scale chiral skyrmions at zero magnetic field $[8,9]$ together with the suppression of the skyrmion Hall effect [10].

Because SAF systems are built in order to have no net magnetization, the observation by most of the standard magnetic imaging techniques represents an important challenge. A solution, that would moreover be interesting for the future potential devices, is to rely on transport measurements instead of an imaging technique. In fact, several contributions to magnetotransport signal in these multilayer systems can be anticipated. Associated to giant magnetoresistance (GMR) effect [11, 12], a significant change in the longitudinal resistance according to the relative orientations of the magnetization in the two FM layers is expected. The GMR is classically expressed as the resistivity change $\Delta \rho_{x x}^{(G)} \propto-\boldsymbol{m}_{1} \cdot \boldsymbol{m}_{2}$, with $\boldsymbol{m}_{i}$ the magnetization in the $i$-th magnetic layer. In multilayers with PMA, which often host one or several non-magnetic heavy metal layers, the total resistance can also change depending on the angle between the magnetization direction $\boldsymbol{m}$ of the FM and the spin polarization $\boldsymbol{\sigma}^{\boldsymbol{s}}$ due to the spin Hall effect in the heavy metal layer(s) [13-17]. Up to present knowledge, such magnetoresistive effect can have different origins depending on the considered materials. In any case, the associated resistivity change can be phenomenologically described by the expression given for spin Hall magnetoresistance [14, 15], according to which $\Delta \rho_{x x}^{(S)} \propto-m_{y}^{2}$ (see axis orientations in Fig. 1). For this reason, hereafter we refer to such contribution as spin Hall magnetoresistance (SMR). This effect differs from anisotropic magnetoresistance (AMR) [18], characterized by a variation of the resistance of the magnetic metals according to the angle between $\boldsymbol{m}$ and the electric current direction $\boldsymbol{J}$, which implies a resistivity change $\Delta \rho_{x x}^{(A)} \propto-m_{x}^{2}$. As for when transverse magnetotransport is concerned, it is expected to be dominated by the Anomalous Hall Effect (AHE) [19], which directly reveals how the average out-of-plane magnetization evolves, according to transverse resistivity $\Delta \rho_{x y} \propto m_{z}$.

All these contributions are mixed together and combine into the accessible magnetotransport information. In this letter, the objective is to demonstrate how the different contributions to the magnetoresistance (MR) listed above can be disentangled, and used to electrically detect non-collinear magnetic chiral textures. The term magnetic chiral textures gathers chiral DWs, spin-spirals and/or skyrmions, in SAF systems and more generally in any multilayers composed of a stacking of a ferromagnetic film (FM) and non magnetic ones (NM). In this study, we focus on the case of detecting spin spiral. Such textures in which the magnetization continuously rotates [9] can be found in SAF stacking having a negligeable outof plane anistoropy but a non zero interfacial DMI. They are characterized by a balanced proportion of parts with in-plane and out-of-plane magnetization, as well as by possibly different relative orientations of the magnetization in the two FM layers, indeed corresponding to the most general case. We show that these textures (as well 


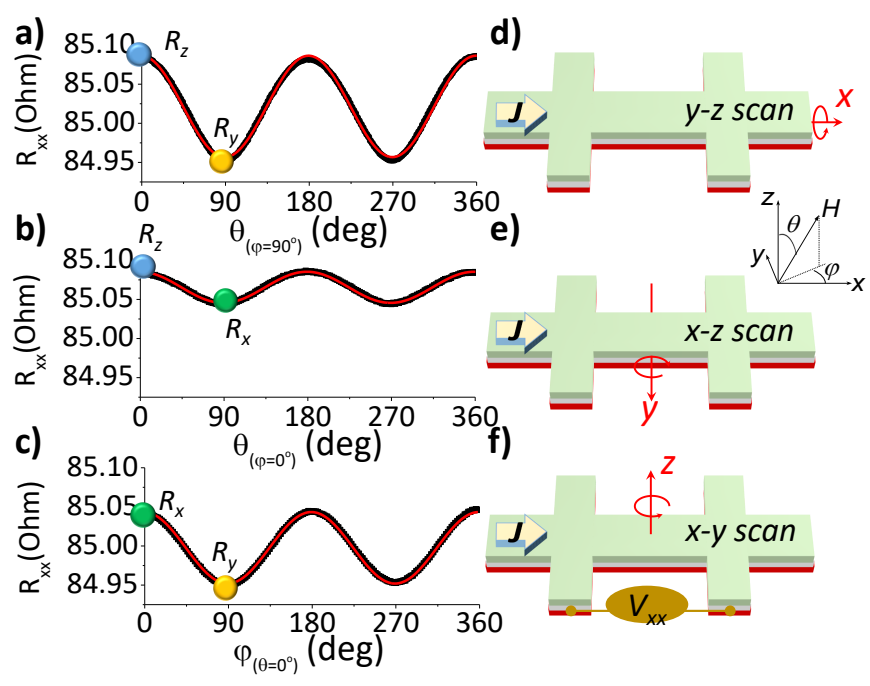

FIG. 1. (a-c) Angular evolution of the longitudinal resistance $R_{x x}$ obtained by rotating the patterned sample in the three main-planes as sketched in (d-f), respectively, while applying a fixed magnetic field of $\mu_{0} H=500 \mathrm{mT}$. The magnetic field is large enough to saturate the FM layers in the three main directions and guarantee that the magnetization follows uniformly the field direction. The dots in (a-c), labelled $R_{i}$, indicate positions at which the FM layers are saturated along the $i^{t h}$ direction. The continuous red lines are the result of fitting the experimental data with the expression of the longitudinal resistivity as given in Eq. (1).

as any other texture with less complexity) can be easily characterized by transport measurements sensitive to all contributions.

The SAF under study for the magneto-electrical characterization has been deposited by magnetron sputtering on a thermally oxidized silicon substrate, and has been subsequently patterned into Hall bars by ion beam etching. The multilayer composition is $\mathrm{Ta}(10) \mid \mathrm{Pt}$ (8) $|\mathrm{Co}(1.47)| \mathrm{Ru}(0.75)|\mathrm{Pt}(0.6)| \mathrm{Co}(1.47)|\mathrm{Ru}(0.75)| \mathrm{Pt}$ (3), where the numbers refer to individual layer thicknesses in $\mathrm{nm}$. In the chosen SAF system, the Co thickness at which the transition from out-of-plane to in-plane anistropy occurs is around $1.47 \mathrm{~nm}$. An advantage for this study is that, for such a thickness, sensitivity, the sensitivity of the electrical transport measurements to magnetoresistive effects is enhanced, as the greater FM/NM ratio allows a large portion of the conduction electrons to flow in the FM layers.

We first characterize contributions to the longitudinal resistivity excluding the GMR, by aligning all moments in the multilayer plane, with the help of an external magnetic field much larger than antiferromagnetic interlayer coupling and DMI fields. By measuring the field evolution of the resistance along the three directions $x, y$ and $z$ (not shown), we verify that a field of $\mu_{0} H=500 \mathrm{mT}$ is large enough to reach magnetic saturation in all the three directions. By then rotating the direction of this external field, we can fully characterize the evolution of $R_{x x}$ with $m_{x}, m_{y}$ and $m_{z}$. In Fig. 1, we show the angular dependence of the longitudinal resistance $R_{x x}$ (recorded at room temperature) when the field is rotated in the three main-planes of the multilayer, i.e. $y-z, x-z$ and $x-y$, as shown in the schematics. This confirms the presence of different mechanisms at the origin of the magnetoresistive transport signal.

The continuous red lines in Fig. 1(a-c) are the results of fitting the experimental data with the expression of the longitudinal resistivity in FM/NM stacks [14] expressed as:

$$
\rho_{x x}=\rho_{0}+\Delta \rho_{S}\left(1-m_{y}^{2}\right)+\Delta \rho_{A}\left(1-m_{x}^{2}\right)
$$

where $\rho_{0}$ is the electrical resistivity, $\Delta \rho_{S}$ and $\Delta \rho_{A}$ are the variations coefficients due to the SMR and AMR, respectively, while the GMR contribution is zero because the two magnetizations are saturated. The $y$ - $z$ scan [Fig. $1(\mathrm{a}, \mathrm{d})]$ shows the largest variation of resistance and a SMR-like behavior. Note that the resistance decreases for $\theta_{\left(\phi=90^{\circ}\right)}=0^{\circ} \rightarrow 90^{\circ}$, and the lowest value is obtained when $\boldsymbol{m} \| \boldsymbol{\sigma}^{s}$. From the fit, we find that SMR contribution dominates, as $\Delta \rho_{S}$ is about $0.15 \%$ and $\Delta \rho_{A}$ about $0.05 \%$. The value for $\Delta \rho_{S}$, obtained from $\left(R_{z}-R_{y}\right) / R_{z}$ with $R_{i}$ the resistance when the FM layers are saturated along the $i^{t h}$ direction, is in agreement with what has been reported for other metallic FM/NM multilayers $[16,17]$. In light of the dependence of $R_{x x}$ with $m_{x}^{2}$ and $m_{y}^{2}$ discussed here, we can anticipate to be sensitive to inplane components of the magnetization even in the case of zero average $m_{x}$ and $m_{y}$. In fact, as shown in Fig. 1(a-b), both SMR and AMR exhibit a large, negative variation of the resistance when the magnetization varies from out-of-plane to in-plane, which can be used to detect the presence of non-collinear magnetic textures. Hereafter, for the sake of clarity, we refer to magnetoresistive contributions from the in-plane component of isotropic magnetic textures as ipMR.

In order to determine other contributions of the magnetization textures to magnetoresistive transport signal (namely, GMR contribution), the magnetization reversal mechanism has to be precisely characterized (simultaneously to the transport measurement) in order, to know the actual magnetic state and then determine the evolution of the magnetic texture in the multilayer [20,21]. For this purpose, the measurement of the Hall resistance $\left(R_{x y}\right)$ is a very appropriate mean, as it is directly linked to the average magnetization along $z$, in particular, when a magnetic field is swept along the out-of-plane direction.

We thus study the evolution of the transverse resistance of the multilayer measured at room temperature as a function of an out-of-plane external field, as shown in Fig. 2(a), thereby revealing how the average out-ofplane magnetization evolves with external field. It shows a smooth variation from one magnetic saturation state to 
the other with opposite orientation. No hysteresis effects appear here and the process is reversible in the full field range. Such smooth variation of $R_{x y}$ reflects the progressive evolution from a parallel alignment of the magnetization in the two FM layers, at large applied fields, to an anti-parallel alignment, at low fields. The absence of signal at remanence indicates that the average $m_{z}$ component is zero due to the formation of balanced spin-spirals in this SAF system [9]. Then, the magnetization process under field is similar to the behavior of an AF system with spin-flop like transition [22-24]: moments with opposite out-of plane orientation are expected to flop into a canted spin state, having opposite in-plane components in the two layers combined with small out-of-plane canting in the direction of the external field. For low applied fields, the magnetization is thus mostly perpendicular to this field, showing the strongest in-plane components, before it starts to fully align with stronger external fields.

In order to confirm our interpretation, we exploit the fact that this behavior is significantly different at low temperature. As shown in Fig. 2(b), the $R_{x y}$ field loop, now measured at $10 \mathrm{~K}$, shows sharp transitions between antiparallel alignment at remanence and parallel alignment under strong enough positive or negative fields. Therefore, we observe a temperature-related transition from continuous evolution, at RT, to stepped magnetization reversal by nucleation of one or a few large domains, when the temperature is decreased. This change of be-
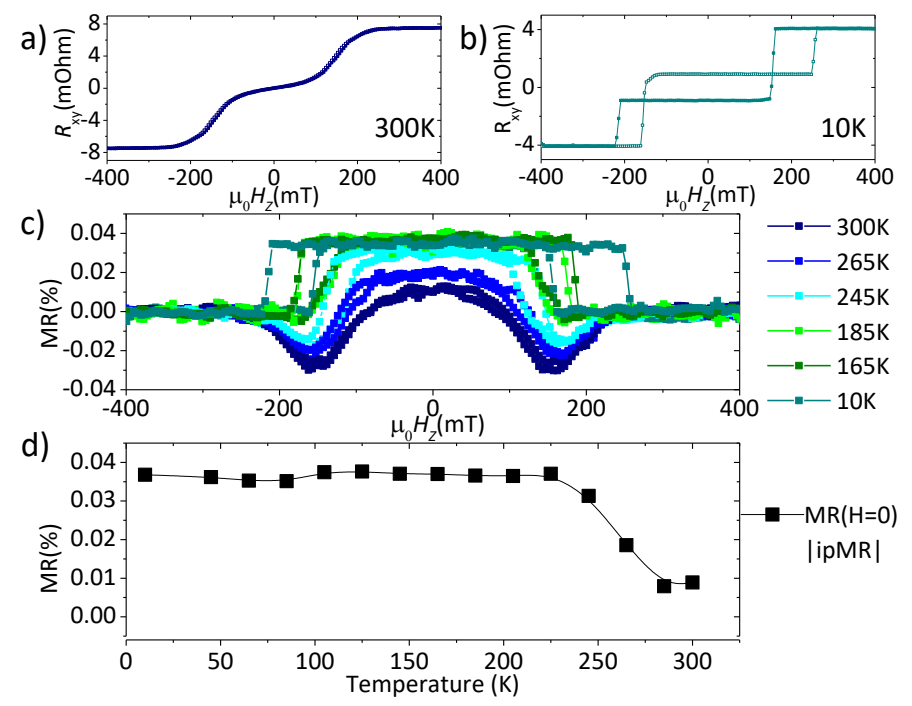

FIG. 2. (a-b) Out-of-plane field dependence of the transverse resistance at two temperatures. The open (filled) data points have been recorded for increasing (decreasing) field. (c) Temperature evolution of the longitudinal resistance $\left(R_{x x}\right)$ as function of out-of-plane magnetic field. (d) Temperature dependence of the $R_{x x}$ at zero field, where the GMR contributions dominate (black dots), and the absolute value of $R_{x x}$ minima, where the ipMR contributions dominates (red dots). havior is mainly due to the enhancement of the perpendicular anisotropy at low temperature, similarly to what has been reported for other SAF systems [25].

In Fig. 2(c), we show the magnetoresistive response at different temperatures, where MR is defined as $\mathrm{MR}=\left[R_{x x}(H)-R_{\|}\right] / R_{\|}$, with $R_{\|}$the longitudinal resistance in the uniform state. Low temperature measurements show sharp transitions, consistent with the sharp transitions also observed in the $R_{x y}$ curves displayed in Fig. 2(b). In this case, the recorded MR arises essentially from GMR signal, as it shows low resistance for parallel alignment of the magnetizations in the two FM layers and large resistance for anti-parallel alignment [11, 12]. The enhancement of out-of-plane anisotropy at low temperature indeed prevents the formation of spin-spirals, by disfavoring any in-plane component for the magnetization, thus canceling ipMR contributions. Looking at the temperature evolution of the curves in Fig. 2(c), we observe however that the zero field MR (as defined here, between saturation and remanence of the magnetization) decreases for increasing temperature. Related to this loss of MR, additional features (a negative variation of the MR) progressively appear in the field range of the nucleation process $(110-200 \mathrm{mT})$, strengthening with increasing temperature. We associate this signal to ipMR, owing to the stabilization of spin-spirals, in which a significant part of magnetic moments are oriented inplane, as the perpendicular anisotropy vanishes towards reaching room temperature. The loss of MR is therefore due to a competition between this negative ipMR contribution and the positive contribution due to GMR. In Fig. 2(d), we display the evolution with temperature of the zero field MR value. Indeed, at remanence the GMR contribution is always present, as at any temperature the remanent state shows antiparallel orientation of the moments everywhere in the two layers. This allows to separate it from the contribution of the ipMR (expected to be zero for strong anisotropy, at $10 \mathrm{~K}$, and maximal for vanishing anisotropy, at $300 \mathrm{~K}$ ). Such assertion is valid provided that temperature variations of $\Delta \rho_{x x}^{(G)}, \Delta \rho_{x x}^{(S)}$ and $\Delta \rho_{x x}^{(A)}$ can be neglected, which is here correct for getting a qualitative understanding. In other words, the reduction of ipMR contribution as the temperature decreases is related to the progressive increase of the size of the magnetic domains, leading to a decrease of the effective fraction of in-plane magnetization components, as indicated by the enhancement of the sharpness of the step variation in $R_{x y}$.

In order to get more insight into the evolution of the overall magnetic texture and of its contribution to electrical properties, we have carried out concomitant measurements of the longitudinal/transverse resistance and imaging by Magnetic Force Microscopy (MFM) at room temperature. The method consisting in combining these two techniques has been demonstrated to be particularly powerful in order to associate magnetotransport signals 


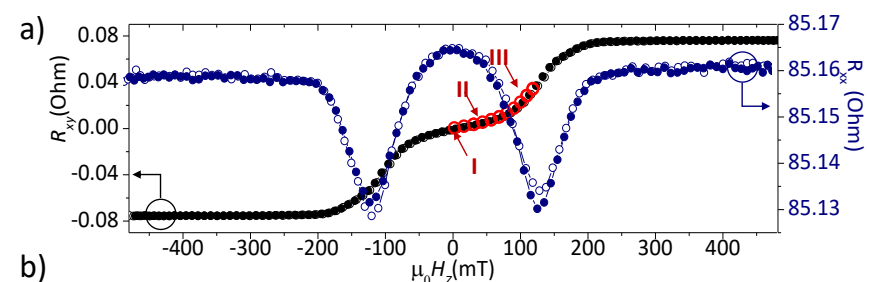

b)

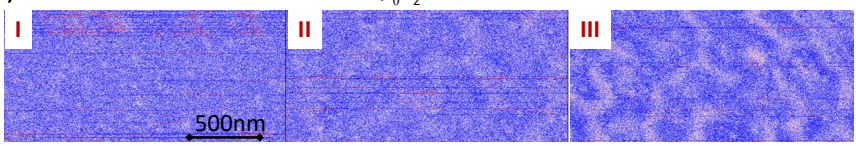

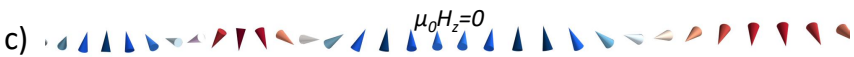

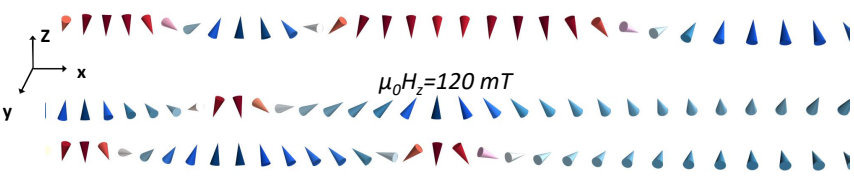

FIG. 3. (a) Evolution of the room-temperature longitudinal and transverse resistance with magnetic field swept along the out-of-plane $(\mathrm{z})$ direction. The red circles are the results of the transverse resistance $\left(R_{x y}\right)$ measured concomitantly with the MFM imaging. (b) MFM phase maps, taken in the same region of the cross-bar region, corresponding to the resistance values indicated in (a). (c) Results of the MuMax3 simulations for magnetization configurations in the FM layers obtained for $\mu_{0} H_{z}=0$ and $120 \mathrm{mT}$.

to the local magnetic configuration, allowing, for example, electrical detection of single magnetic skyrmions with sub-100nm size through transverse resistivity changes [26]. For the present system, room temperature evolution of $R_{x x}$ and $R_{x y}$ with field is displayed in Fig. 3(a). The red circles correspond to $R_{x y}$ measured simultaneously to MFM imaging. This measured transverse resistance, being consistent with previous data, allows to exclude any significant modification of the magnetic texture under the influence of the MFM magnetic tip. In Fig. 3(b), three images are shown among all MFM images recorded, corresponding to the Hall cross region of a lithographed device with a track width of $5 \mu \mathrm{m}$. The MFM data in image I, showing no contrast, demonstrates that at remanence the total magnetization $\boldsymbol{m}_{1}+\boldsymbol{m}_{2}$ has no vertical component anywhere in the track. The stray field arising from compensated magnetic textures is indeed expected to be almost completely cancelled in case of interlayer AF coupling of the magnetization in the two layers [27]. Micromagnetic simulations of the expected magnetic textures with MuMax3 solver [28] using our experimentally determined parameters (more details about the simulations and the parameters used [? ] can be found elsewhere [9]), confirm this interpretation. As shown in Fig. 3(c), the magnetic configuration at zero field corresponds to cycloidal spin-spirals antiferromagnetically coupled between the two FM layers, only giving rise to stray fields below the MFM setup sensitivity, as the magnetic moments everywhere in both FM layers are fully compensated by each other (see top part of Fig. $3(\mathrm{c}))$. We remark that the DMI-induced chirality, together with the AF coupling, are indeed compatible with magnetizations pointing in opposite direction in the FM layers everywhere along the textures. Note that this corresponds to a different case compared to what has been reported by Hellwig et al. in Ref. [29], where the absence of MFM contrast is due to AF coupling between layers with uniform magnetization. When an out-of-plane field is applied, we observe a weak signal appearing in the MFM images [image II of Fig. 3(b)], which further builds up as the magnetic field is further increased [image III of Fig. 3(b)]. When an external magnetic field is applied, it introduces an energy unbalance between parts of the spiral with $m_{z}>0$ and $m_{z}<0$, and thus it causes an enlargement of the portions of the spirals where the magnetization aligns with the field and a compression of the others portions. This phenomenon occurs in addition to previously described spin-flop related canting of the magnetization increasing the proportion of in-plane moments. This is confirmed by the magnetic configuration obtained by micromagnetic simulation under external field, as shown in Fig. 3(c). The out-of plane field perturbs the antiparallel alignment observed at remanence and full magnetic compensation is lost, consistent with the contrast appearing in MFM images. This process thus explains the results of our electrical measurements: the field dependence of the resistance shows a continuous increase in $R_{x y}$ in link with the increase of total magnetic moment aligned along $z$, as expected from AHE, while the evolution of $R_{x x}$ results from antagonist MR effects due to ipMR and GMR.

The connection between the magnetization evolution and the electrical transport properties can even be quantitatively understood, by fitting experimental variations of resistance with external field, based on the magnetic textures determined from micromagnetic simulations, as shown in Fig. 4. The variations of the longitudinal resistance are expressed as $\Delta R_{x x}=R_{x x}-R_{\text {sat }}$ where $R_{\text {sat }}$ is the longitudinal resistance at out-of-plane saturation. The data points are fitted using the maps of $m_{x}, m_{y}$ and $m_{z}$ in each layer, as determined by micromagnetic calculations, allowing only two phenomenological parameters $a$ and $b$ entering in $\Delta R_{x x}=-a \boldsymbol{m}_{i p}^{2}+b(1-\cos (\alpha))$, $\alpha$ being the angle between $\boldsymbol{m}_{1}$ and $\boldsymbol{m}_{2}$. We note that the experimental results are well reproduced, and notably the two main features of MR: the negative variation of resistance at intermediate fields and the difference between remanence and saturation. We attribute the latter mainly to the monotonous evolution of $\cos (\alpha)$ in the GMR term, and the former to the field dependence of the in-plane magnetization $\left(m_{i p}^{2}\right)$. The fit (red dotted curve) shown in Fig. 4 provides $a=7.8 \times 10^{-2}$ $\mathrm{Ohm}$ and $b=3.1 \times 10^{-3} \mathrm{Ohm}$. As a key result, the value of $a$ deduced from this fit is matching with the 


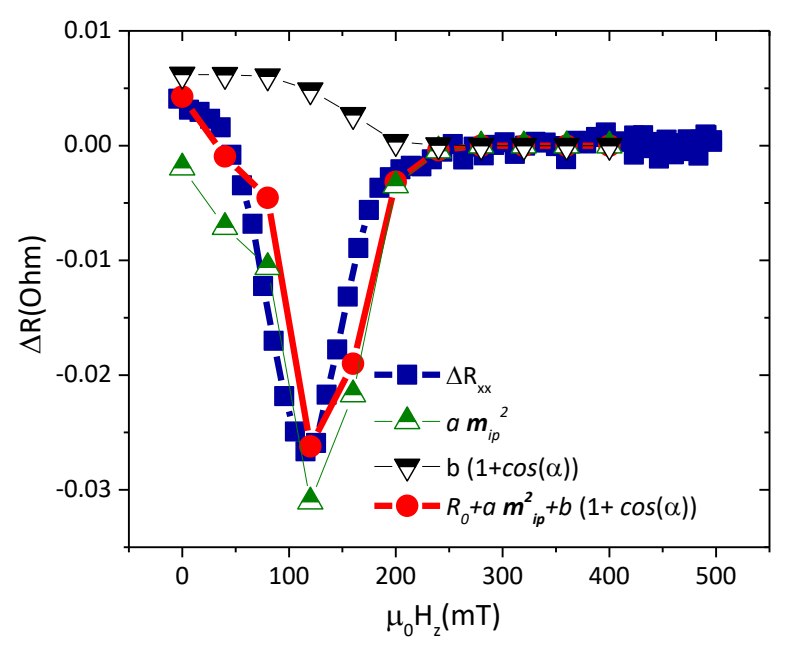

FIG. 4. Comparative results of simulation vs. MR measured at room temperature. The contribution of the in-plane moments (upward green triangles) and the relative magnetization in two layers (downward black triangles) are fitted (red dots) to the experimental data (blue squares).

ipMR determined from our initial characterization, with $R_{z}-\left(R_{x}+R_{y}\right) / 2=0.08 \mathrm{Ohm}$ in Fig. 1 . In view of their agreement with the micromagnetic simulations, the experimental magnetotransport results allow to draw a comprehensive picture of the evolution of the magnetization texture in our system as a function of the applied magnetic field.

In conclusion of the present study, electrical transport characterization thus turns out to provide crucial information about the reversal mechanisms between different magnetic configurations in SAF multilayers with or without perpendicular anisotropy. We have demonstrated that the combination of the different magnetization components gives rise to a competition between several magnetoresistive effects of different origins that can be grouped, respectively, into GMR and ipMR. These phenomena allow us, relying on electrical transport characterization, to extract qualitative information about magnetic properties in the system, and quantitative information about the relative proportions of all magnetization components in the stabilized magnetic textures. This establishes electrical transport measurements as a very suitable tool to detect spin textures in a broad class of multilayers, extending previous possibilities towards the detection of isolated magnetic domains, spin-spirals or even skyrmions in SAF systems, with applications to their easier characterization, and probing [26, 30] in race-track like devices [4].

Financial support from the Agence Nationale de la Recherche, France, under grant agreement No. ANR17-CE24-0025 (TOPSKY), the DARPA TEE program, through grant MIPR No. HR0011831554 and the Horizon2020 Framework Programme of the European Commission, under FET-Proactive Grant agreement No. 824123 (SKYTOP) and FET-Open grant agreement No. 665095 (MAGicSky) is acknowledged.

* davide.maccariello@cnrs-thales.fr

† vincent.cros@cnrs-thales.fr

[1] S. S. P. Parkin, N. More, and K. P. Roche. Oscillations in exchange coupling and magnetoresistance in metallic superlattice structures: $\mathrm{Co} / \mathrm{Ru}, \mathrm{Co} / \mathrm{Cr}$, and $\mathrm{Fe} / \mathrm{Cr}$. Physical Review Letters, 64(19):2304-2307, 1990.

[2] S. S. P. Parkin and D. Mauri. Spin engineering: Direct determination of the Ruderman-Kittel-Kasuya-Yosida far-field range function in ruthenium. Physical Review B, 44(13):7131-7134, 1991.

[3] A. Fert, V. Cros, and J. Sampaio. Skyrmions on the track. Nature Nanotechnology, 8(3):152-156, 2013.

[4] S. S. P. Parkin and S. H. Yang. Memory on the racetrack. Nature Nanotechnology, 10(3):195-198, 2015.

[5] I. Dzyaloshinsky. A thermodynamic theory of weak ferromagnetism of antiferromagnetics. Journal of Physics and Chemistry of Solids, 4(4):241-255, 1958.

[6] T. Moriya. Anisotropic Superexchange Interaction and Weak Ferromagnetism. Physical Review, 120(1):91-98, 1960.

[7] A. Fert. Magnetic and Transport Properties of Metallic Multilayers. Materials Science Forum, 1990.

[8] F. Büttner, I. Lemesh, and G. S.D. Beach. Theory of isolated magnetic skyrmions: From fundamentals to room temperature applications. Scientific Reports, 8(1):1-12, 2018.

[9] W. Legrand, D. Maccariello, F. Ajejas, W. Akhtar, S. Collin, K. Bouzehouane, N. Reyren, V. Jaques, V. Cros, and A. Fert. Room-temperature stabilization of magnetic skyrmions in synthetic antiferromagnets. Nature Materials, doi.org/10.1038/s41563-0190468-3:, Sept. 2, 2019.

[10] Xichao Zhang, Yan Zhou, and Motohiko Ezawa. Magnetic bilayer-skyrmions without skyrmion Hall effect. $\mathrm{Na}$ ture Communications, 7:10293, 2016.

[11] M. N. Baibich, J. M. Broto, A. Fert, F. Nguyen Van Dau, F. Petroff, P. Etienne, G. Creuzet, A. Friederich, and J. Chazelas. Giant Magnetoresistance of (001)Fe/(001)Cr Magnetic Superlattices. Physical Review Letters, 61(21):2472-2475, 1988.

[12] P. Grünberg, R. Schreiber, Y. Pang, M. B. Brodsky, and H. Sowers. Layered Magnetic Structures: Evidence for Antiferromagnetic Coupling of Fe Layers across Cr Interlayers. Physical Review Letters, 57(19):2442-2445, 1986.

[13] A. Kobs, S. Hee, W. Kreuzpaintner, G. Winkler, D. Lott, P. Weinberger, A. Schreyer, and H. P. Oepen. Anisotropic Interface Magnetoresistance in Pt / Co / Pt Sandwiches. Physical Review Letters, 106(21), 2011.

[14] Y.-T. Chen, S. Takahashi, H. Nakayama, M. Althammer, S. T. B. Goennenwein, E. Saitoh, and G. E. W. Bauer. Theory of spin Hall magnetoresistance. Physical Review $B, 87(14), 2013$.

[15] H. Nakayama, M. Althammer, Y.-T. Chen, K. Uchida, Y. Kajiwara, D. Kikuchi, T. Ohtani, S. Geprgs, M. Opel, 
S. Takahashi, R. Gross, G. E. W. Bauer, S. T. B. Goennenwein, and E. Saitoh. Spin Hall Magnetoresistance Induced by a Nonequilibrium Proximity Effect. Physical Review Letters, 110(20):206601, 2013.

[16] C. O. Avci, K. Garello, A. Ghosh, M. Gabureac, Santos F. Alvarado, and P. Gambardella. Unidirectional spin Hall magnetoresistance in ferromagnet/normal metal bilayers. Nature Physics, 11(7):570-575, 2015.

[17] C. O. Avci, K. Garello, J. Mendil, A. Ghosh, N. Blasakis, M. Gabureac, M. Trassin, M. Fiebig, and P. Gambardella. Magnetoresistance of heavy and light metal/ferromagnet bilayers. Applied Physics Letters, 107(19):192405, 2015.

[18] T. McGuire and R. Potter. Anisotropic magnetoresistance in ferromagnetic $3 \mathrm{~d}$ alloys. IEEE Transactions on Magnetics, 11(4):1018-1038, 1975.

[19] N. Nagaosa, J Sinova, S Onoda, A. H. MacDonald, and N. P. Ong. Anomalous Hall effect. Reviews of Modern Physics, 82(2):1539-1592, 2010.

[20] P. Perna, C. Rodrigo, M. Muoz, J. L. Prieto, A. Bollero, D. Maccariello, J. L. F. Cuado, M. Romera, J. Akerman, E. Jimnez, N. Mikuszeit, V. Cros, J. Camarero, and R. Miranda. Magnetization reversal signatures in the magnetoresistance of magnetic multilayers. Physical Review B, 86(2):024421, 2012.

[21] P. Perna, D. Maccariello, C. Rodrigo, J. L. F. Cuado, M. Muoz, J. L. Prieto, M. A. Niño, A. Bollero, J. Camarero, and R. Miranda. Direct experimental determination of the anisotropic magnetoresistive effects. Applied Physics Letters, 104(20):202407, 2014.

[22] B. Dieny, J. P. Gavigan, and J. P. Rebouillat. Magnetisation processes, hysteresis and finite-size effects in model multilayer systems of cubic or uniaxial anisotropy with antiferromagnetic coupling between adjacent ferromagnetic layers. Journal of Physics: Condensed Matter, 2(1):159, 1990.
[23] K. Ounadjela, D. Muller, A. Dinia, A. Arbaoui, P. Panissod, and G. Suran. Perpendicular anisotropy and antiferromagnetic coupling in $\mathrm{Co} / \mathrm{Ru}$ strained superlattices. Physical Review B, 45(14):7768-7771, 1992.

[24] S. Hamada, K. Himi, T. Okuno, and K. Takanashi. MFM observation of perpendicular magnetization and antiferromagnetically coupled domains in $\mathrm{Co} / \mathrm{Ru}$ superlattices. Journal of Magnetism and Magnetic Materials, 240(1):539-542, 2002.

[25] O. Hellwig, A. Berger, J. B. Kortright, and E. E. Fullerton. Domain structure and magnetization reversal of antiferromagnetically coupled perpendicular anisotropy films. Journal of Magnetism and Magnetic Materials, 319(1-2):13-55, 2007.

[26] D. Maccariello, W. Legrand, N. Reyren, K. Garcia, K. Bouzehouane, S. Collin, V. Cros, and A. Fert. Electrical detection of single magnetic skyrmions in metallic multilayers at room temperature. Nature Nanotechnology, 13(3):233-237, 2018.

[27] N. S. Kiselev, I. E. Dragunov, V. Neu, U. K. Rler, and A. N. Bogdanov. Theoretical analysis of magnetic force microscopy contrast in multidomain states of magnetic superlattices with perpendicular anisotropy. Journal of Applied Physics, 103(4):043907, 2008.

[28] A. Vansteenkiste, J. Leliaert, M. Dvornik, M. Helsen, F. Garcia-Sanchez, and B. Van Waeyenberge. The design and verification of MuMax3. AIP Advances, 4(10):107133, 2014.

[29] O. Hellwig, A. Berger, and E. E. Fullerton. Domain walls in antiferromagnetically coupled multilayer films. Physical Review Letters, 91(19):3-6, 2003.

[30] M. Hayashi, L. Thomas, R. Moriya, C. Rettner, and S. S. P. Parkin. Current-Controlled Magnetic DomainWall Nanowire Shift Register. Science, 320(5873):209$211,2008$. 